Gut and Liver, Vol. 10, No. 1, January 2016, pp. 63-68

\title{
Negative Biopsy after Referral for Biopsy-Proven Gastric Cancer
}

\author{
Chung Hyun Tae* ${ }^{\star}$, Jun Haeng Lee*, Byung-Hoon Min*, Kyoung-Mee Kim ${ }^{\ddagger}$, Poong-Lyul Rhee*, and Jae J. Kim* \\ *Department of Medicine, Samsung Medical Center, Sungkyunkwan University School of Medicine, ${ }^{\dagger}$ Department of Internal Medicine, Ewha \\ Womans University School of Medicine, and ${ }^{\ddagger}$ Department of Pathology, Samsung Medical Center, Sungkyunkwan University School of \\ Medicine, Seoul, Korea
}

Background/Aims: Repeat endoscopy with biopsy is often performed in patients with previously diagnosed gastric cancer to determine further treatment plans. However, biopsy results may differ from the original pathologic report. We reviewed patients who had a negative biopsy after referral for gastric cancer. Methods: A total of 116 patients with negative biopsy results after referral for biopsy-proven gastric cancer were enrolled. Outside pathology slides were reviewed. Images of the first and second endoscopic examinations were reviewed. We reviewed the clinical history from referral to the final treatment. Results: Eighty-eight patients (76\%) arrived with information about the lesion from the referring physician. Among 96 patients with available outside slides, the rate of interobserver variation was $24 \%$. Endoscopy was repeated at our institution; 85 patients (73\%) were found to have definite lesions, whereas 31 patients (27\%) had indeterminate lesions. In the group with definite lesions, 71\% of the lesions were depressed in shape. The most common cause of a negative biopsy was mistargeting. In the group with indeterminate lesions, 94\% had insufficient information. All patients with adequate follow-up were successfully treated based on the findings in the follow-up endoscopy. Conclusions: A negative biopsy after referral for biopsy-proven gastric cancer is mainly caused by mistargeting and insufficient information during the referral. (Gut Liver 2016;10:63-68)

Key Words: Stomach; Neoplasms; Biopsy; Gastroscopy

\section{INTRODUCTION}

Gastric cancer is one of the most common cancers in Korea. ${ }^{1}$ It's age-standardized incidence is 62.9 per 100,000 person-years for men and 41.7 for women in 2011. ${ }^{1}$ Upper endoscopy has been widely performed in outpatient clinics or health promo- tion centers for the purpose of early detection of gastric cancer in Korea. ${ }^{2}$ Therefore, many gastric cancers are detected at their very early stages.

When a patient with gastric cancer confirmed by biopsies was referred to tertiary hospitals, most physicians repeat endoscopic examinations with biopsies before the final decision for the treatment. Although not frequent, biopsy result at the referred hospital is negative for malignancy in some patients. It makes great confusion for doctors and patients alike. To the best of our knowledge, there is no literature on this challenging clinical situation. We retrospectively reviewed 116 patients with negative biopsy after referral for biopsy-proven gastric cancer.

\section{MATERIALS AND METHODS}

\section{Study population}

When patients were referred with pathologically proven gastric cancer diagnosis, we perform upper endoscopic examinations with biopsies. All endoscopy was performed by expert endoscopists at our institution. In some patients, the first biopsies at our institution were negative for malignancy. Using a collected patient database of a single physician (J.H.L.), we found 116 consecutive patients with negative biopsy result after referral for biopsy-proven gastric cancer from July 2003 to April 2010. In a majority of enrolled patients, additional endoscopy with biopsies was performed. This study was approved by the Institutional Review Board of the Samsung Medical Center.

\section{Analysis of outside medical information}

At the time of referral, we tried to get as much information as possible based on the outside medical information from referring physicians, such as endoscopic report and images, pathologic report, and hematoxylin and eosin (H\&E)-stained pathologic slides. Based on the collected data, we analyzed quality

\footnotetext{
Correspondence to: Jun Haeng Lee

Department of Medicine, Samsung Medical Center, Sungkyunkwan University School of Medicine, 81 Irwon-ro, Gangnam-gu, Seoul 06351, Korea

Tel: +82-2-3410-3409, Fax: +82-2-3410-6983, E-mail: stomachlee@gmail.com

Received on July 25, 2014. Revised on October 22, 2014. Accepted on November 3, 2014. Published online May 13,2015

pISSN 1976-2283 eISSN 2005-1212 http://dx.doi.org/10.5009/gnl14281

@) This is an Open Access article distributed under the terms of the Creative Commons Attribution Non-Commercial License (http://creativecommons.org/licenses/by-nc/4.0) which permits unrestricted non-commercial use, distribution, and reproduction in any medium, provided the original work is properly cited.
} 
and quantity of the outside medical information as follows. We evaluated the information about location and shape, and divided the cases into three categories such as sufficient, insufficient or absent. If the outside endoscopic report and images has all of the information about location and shape, it was considered as sufficient. If there was no information, it was considered as absent.

To determine the rate of interobserver variation, the outside H\&E-stained pathologic slides were reviewed by experienced pathologists at our institution, and compared to the outside pathologic reports.

\section{Analysis of our first endoscopy report and images}

We reviewed the endoscopic reports and images of the first endoscopy done after referral at our institution. We evaluated the definiteness of the lesion in terms of the location and shape, and divided the cases into the two groups: the definite lesion group ( $n=85)$ and the indefinite lesion group $(n=31)$. The diagnosis of definite lesion group was established if the primary lesion of interest during our first endoscopy was distinct, so it was not different from the reported biopsy site at outside endoscopy. If the lesion was indistinct, so it was different from the reported biopsy site of outside endoscopy, it was classified the indefinite lesion. In the definite lesion group, we tried to search the possible reason of negative biopsy in the first endoscopy. In the indefinite lesion group, we tried to find the reason why we couldn't locate the lesion. The number of biopsy fragments was counted to assess the extent of biopsy sampling.

\section{Clinical history of $\mathbf{1 1 6}$ patients}

We followed the clinical history for each patient from referral to the ultimate treatment. We reviewed data as follows: total duration from referral to ultimate treatment; the number and intervals of repeated endoscopies; biopsy results on the lesion at every time; selection of ultimate treatment modality; and final pathologic diagnosis known after ultimate treatment.

\section{Statistical analysis}

All of the statistics were performed using SPSS version 20.0 (IBM Corp., Armonk, NY, USA). Continuous data are presented as mean \pm standard deviation. Categorical data are presented as percentages.

\section{RESULTS}

\section{Characteristics of the patients}

The enrolled patients included 90 men and 26 women, with mean age of $57.5 \pm 12.0$ years (range, 19 to 88 years). The mean interval between the initial outside endoscopy and the first endoscopy at our institution was $14.8 \pm 6.1$ days. The clinical diagnoses based on outside pathologic reports from referring physicians were as follows: 29 well-differentiated adenocarcino- mas, 31 moderately-differentiated adenocarcinomas, six poorlydifferentiated adenocarcinomas, 30 signet ring cell carcinomas, 15 adenocarcinomas with no mention of differentiation, and five suspicious malignancies.

\section{Availability of the outside medical information}

Based on the collected outside data, the quality of information including location and morphology was classified into sufficient, insufficient and absent. More than two-thirds of patients ( $n=88,75.9 \%$ ) came to our hospital with at least one information about the location and morphology at the initial endoscopy. Of the outside data, outside endoscopic images, such as Polaroid films or electronic image files were available in only half of the patients ( $n=56,48.3 \%)$. In five of 56 cases with outside endoscopic images, we found that the morphology of the lesion came to have discrepancy compared with outside endoscopic image. In four of five cases, shallow depressed lesions such as early gastric cancer (EGC) type IIc have changed into deep or widen ulceration after the range of 12 to 15 days. The range of biopsy frequency was six to 11 ; these changes may results in massive biopsies at outside endoscopy. In one case showing EGC type III, the lesion has changed into the linear scar with uneven reddish regeneration of the background mucosa after 6 weeks; these changes may results the natural history of gastric cancer.

Review of the outside H\&E-stained pathologic slides was possible in 96 cases (82.8\%).

\section{Analysis of our first endoscopy report and image}

Based on the first endoscopy performed at our institution, 85 cases (73.3\%) were classified as definite lesions, and 31 cases (26.7\%) as indefinite lesions (Fig. 1).

In the endoscopy of the definite lesion group, 76 (89.4\%) were classified as EGC-like lesions and nine lesions (10.6\%) as advanced gastric cancer (AGC)-like lesions. In the 76 EGC-like lesions, 55 lesions (72.4\%) had a depressed shape, such as EGC type IIc $(n=30)$ or III $(n=25)$. In the nine AGC-like lesions, five lesions had a depressed shape, such as Bormann type II $(n=2)$ or III $(n=3)$. We tried to determine the probable reasons of negative biopsy in the repeat endoscopy. In some cases, there was some probable reasons, such as Bormann type IV ( $\mathrm{n}=4,4.8 \%)$, possibility of removed by previous biopsy ( $n=4,4.8 \%)$, misleading outside information about cancer location $(n=2,2.4 \%)$, and misinterpretation of temporary pathologic report $(n=1,1.2 \%)$. The only explanation for cases without probable reason was mistargeting $(\mathrm{n}=74,87.0 \%)$. The number of biopsy fragments was $5.0 \pm 1.6$ in the definite lesion group.

When final endoscopic images were retrospectively analyzed, 28 lesions of indefinite lesion group were classified as EGC and the three lesions as invisible gastric cancer. In the 28 EGC-like lesions, 14 lesions (50.0\%) had a flat shape of EGC IIb (Fig. 1). When we evaluated the possible reason why we could not find the lesion in the indefinite lesion group, the outside endoscopic 
A

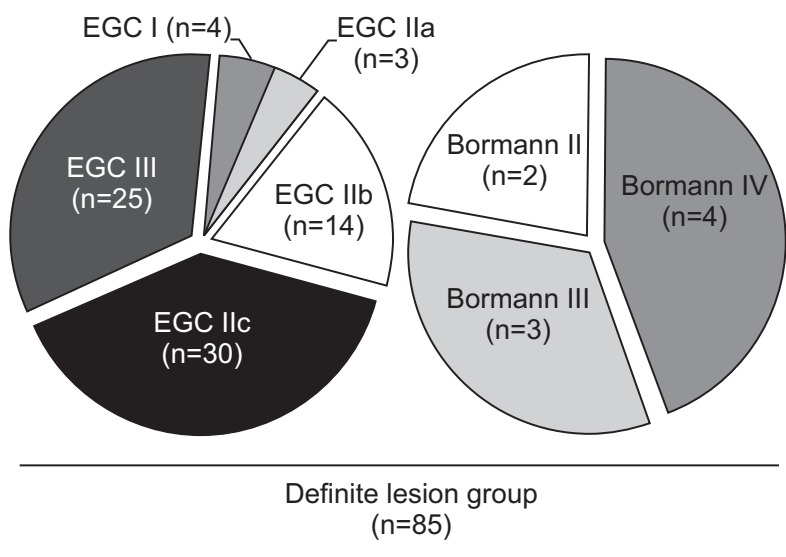

B

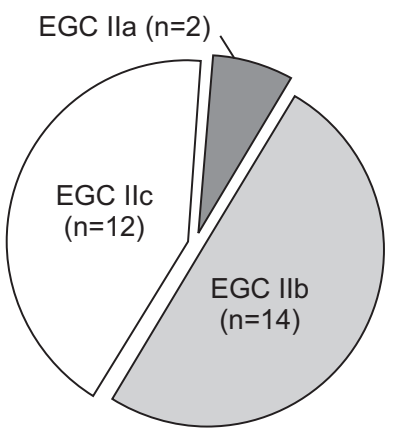

Indefinite lesion group $(n=28)$
Fig. 1. Diagram of morphological classification in 85 definite (A) and 28 indeterminate (B) lesions, except for three cases with gastric cancer that could not be visualized. In the definite lesion group, the most common endoscopic morphology was a depressed lesion, such as early gastric cancer (EGC) type IIc and EGC type III. Flat lesions of EGC type IIb were most common in the indeterminate lesion group.
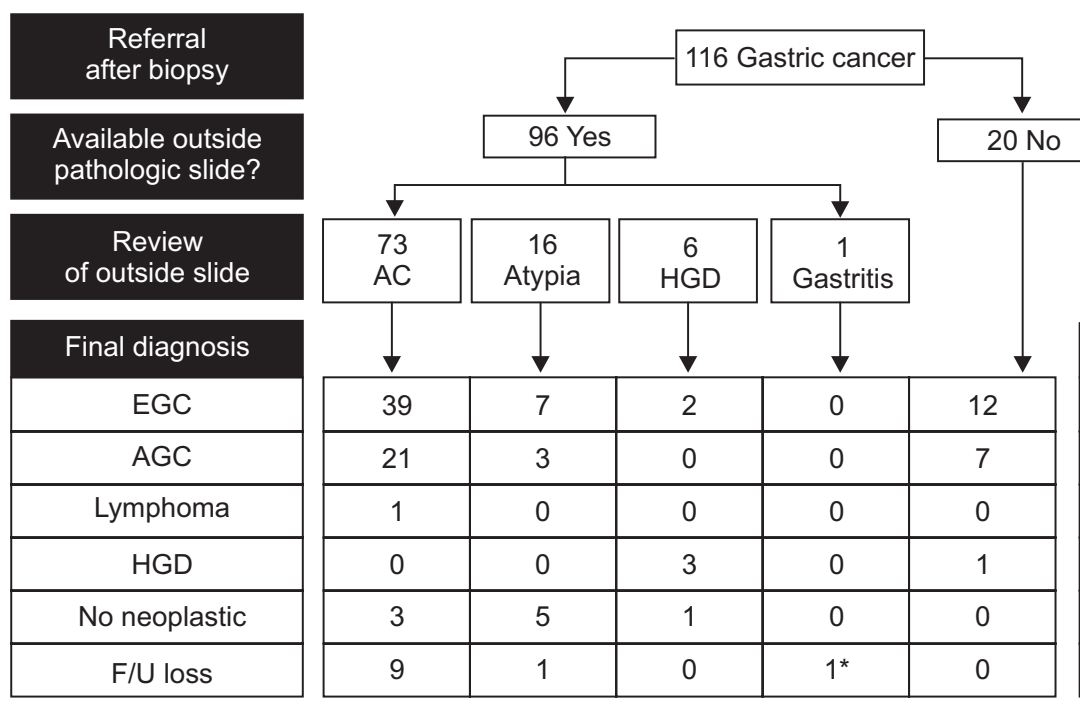

Total

60

31

31

1

4

9

11
Fig. 2. In-house pathologic review and final diagnosis from resected gastric specimen. Of the 96 patients with available outside pathology slides, interobserver variation was found in 23 patients (24.0\%).

AC, adenocarcinoma; HGD, highgrade dysplasia; EGC, early gastric cancer; AGC, advanced gastric cancer; F/U loss, loss to follow-up. *A representative case that was referred to our institution with advanced gastric cancer 4 years after loss to follow-up (Fig. 5). reports or images of 29 patients (77.4\%) were classified into insufficient or absent. Seventeen patients (54.8\%) did not have the outside pathological slides.

\section{Interobserver variation among the pathologists}

When outside pathologic slides were reviewed in 96 cases, 73 cases (76\%) were adenocarcinoma, 17 cases (17.7\%) were nonneoplastic lesions such as atypical gland $(n=16)$ or chronic gastritis $(n=1)$, and six cases (6.3\%) were high grade dysplasia (Fig. 2). Despite adenocarcinoma in the outside slide review by our pathologists, there was no cancer in the final diagnosis of three patients. Repeated follow-up endoscopic examinations were done in two patients for 36 and 11 months, respectively. In one patient, subtotal gastrectomy was done, but there was no evidence of malignancy in the surgical resected specimen.

\section{Clinical courses}

Among 85 cases in the definite lesion group, 15 cases were treated with endoscopic resection or surgery after the first negative biopsy (Fig. 3). The second endoscopic biopsy results of the remaining 70 cases were adenocarcinomas in 40 cases, inflammation in 28 cases, and high grade dysplasia in two cases. A third or fourth endoscopy was done in 14 cases (16.5\%). The mean duration between the referral and the ultimate treatment was $40.1 \pm 20.0$ days (Fig. 3).

Among 31 cases in the indefinite lesion group, treatment was not done after the first biopsy. It was because the lesion could not be clearly localized. Adenocarcinoma was confirmed in the second to fifth endoscopic biopsy in 15 cases (Fig. 4). The mean duration between the referral and the ultimate treatment was $43.3 \pm 19.6$ days except three cases showing the invisible gastric cancer (Fig. 4).

\section{A case of AGC after loss to follow-up}

A 42-year-old female patient visited our institution with a finding of a shallow depressed lesion with hyperemia at greater curvature side of mid-body on outside endoscopic report. However, outside endoscopic picture and pathologic slide were not available. Because we could not find the lesion at our endoscopic examinations, we recommended the patient to bring outside 


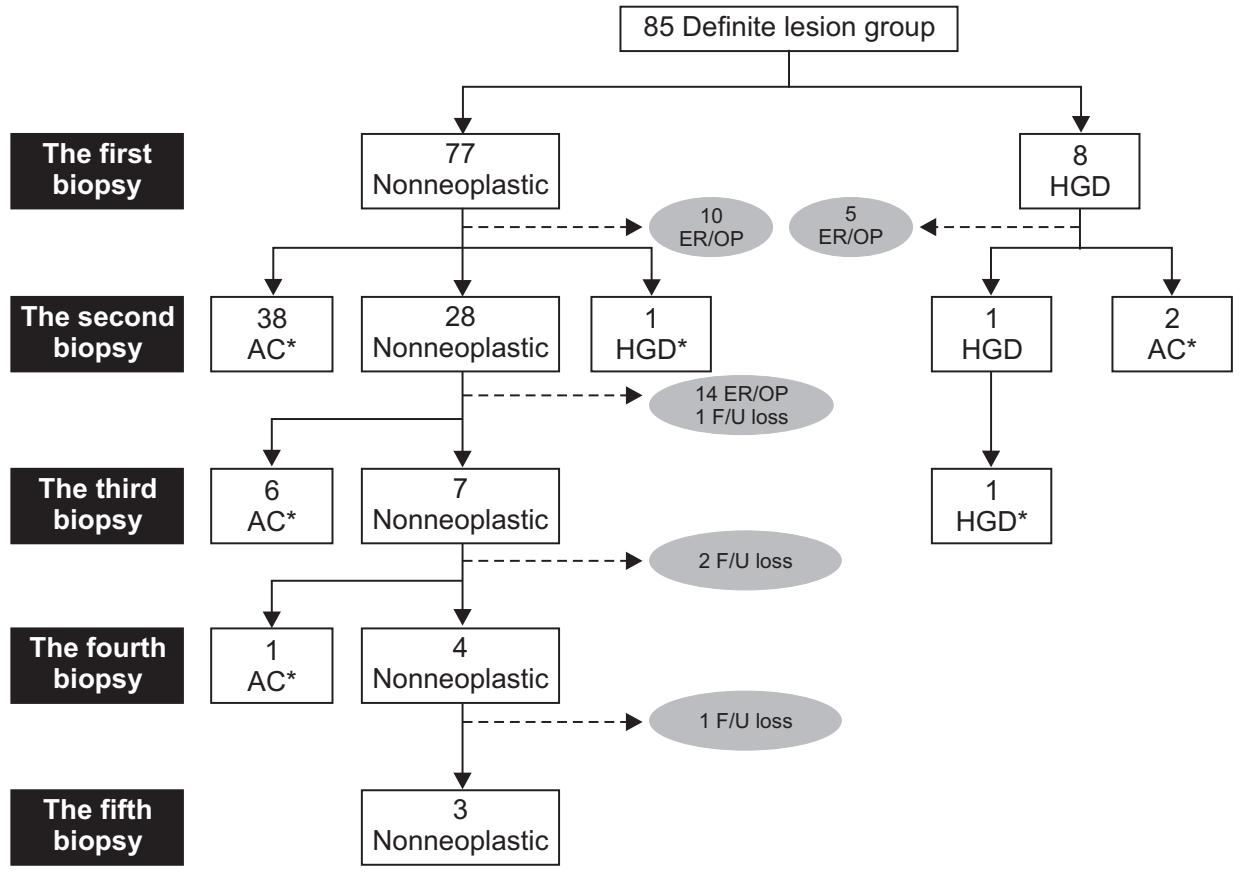

Fig. 3. Flow chart of the definite lesion group. This flow chart shows the results of biopsies at our institution from referrals with biopsy-proven gastric cancer in the definite lesion group.

HGD, high-grade dysplasia; ER/OP, endoscopic resection or operation; $\mathrm{AC}$, adenocarcinoma; F/U, followup. *These patients underwent endoscopic resection or operation following the biopsies.

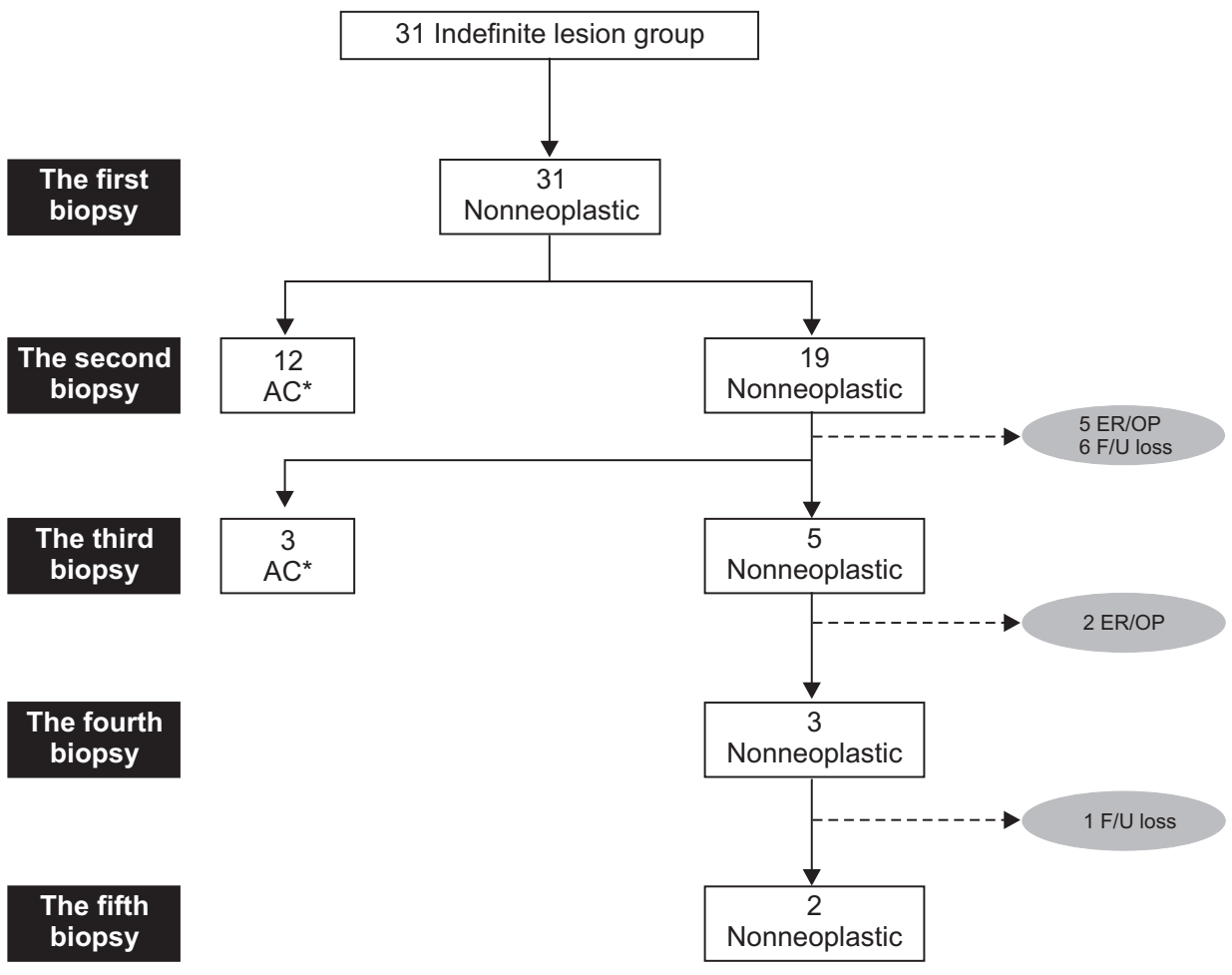

Fig. 4. Flow chart of the indeterminate lesion group. This flow chart shows the results of biopsies at our institution from referrals of patients with biopsy-proven gastric cancer in the indeterminate lesion group. $\mathrm{AC}$, adenocarcinoma; ER/OP, endoscopic resection or operation; $\mathrm{F} / \mathrm{U}$, follow-up. *These patients underwent endoscopic resection or operation following biopsy.

endoscopic pictures and pathologic slides. However, the patent was lost to follow-up. Four years later, the patient was referred to our institution with AGC of Bormann type IV (Fig. 5).

\section{DISCUSSION}

The questions addressed by the present study were (1) how we manage the patient with negative biopsy on the first endoscopy after referral for gastric cancer and (2) what kind of effort is required to prevent the negative biopsy.

In our study, the lesion showing negative biopsy despite a clear lesion had mostly ulcerative morphology (Fig. 1). Earlier studies reported that diagnostic accuracy of endoscopic gastric biopsy can differ by the biopsy site in the ulcerative tumor., The positive biopsy rate was increased to $95 \%$ when the results from the rim and the slough were combined in ulcerative le- 

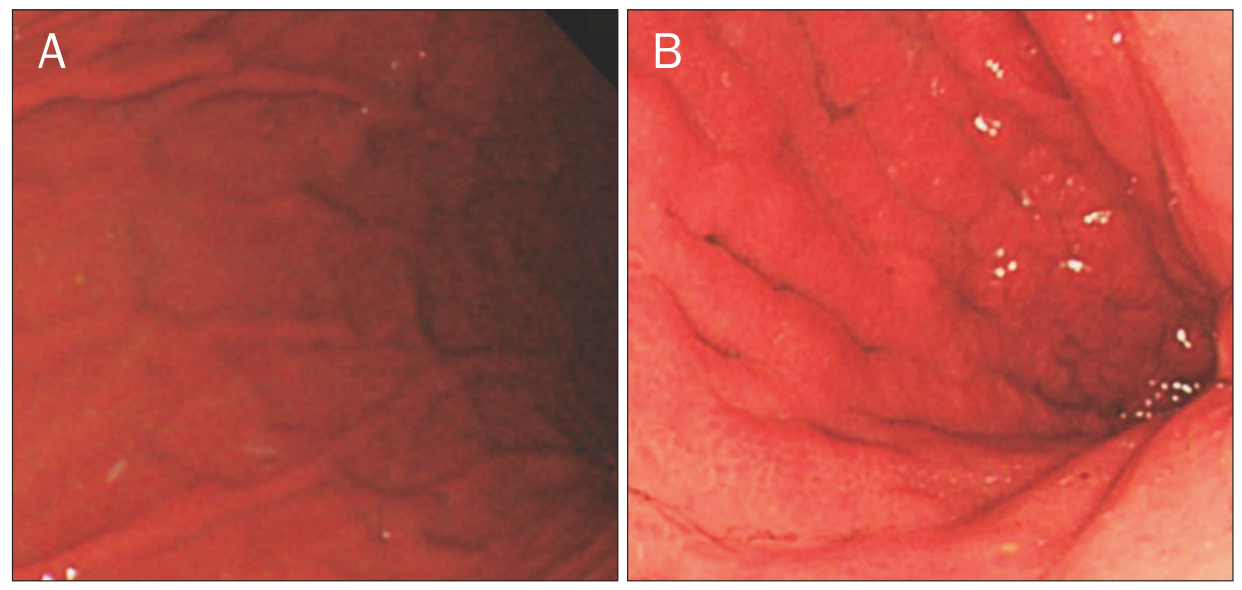

Fig. 5. A representative case showing the importance of robust followup planning. (A) At the time of referral from a local institution, we could not locate the suspicious malignant lesion at the greater curvature of the mid-body in our first endoscopy. (B) Four years after being lost to followup, the patient presented to our hospital with Bormann type IV cancer.

Table 1. Availability and the Quality Analysis of Outside Medical Information

\begin{tabular}{cc}
\hline Outside information source & No. (\%) \\
\hline Endoscopic report & $76(65.6)$ \\
Sufficient* $^{*}$ & $12(10.3)$ \\
Insufficient $^{\dagger}$ & $28(24.1)$ \\
Absent & \\
Endoscopic images & $56(48.3)$ \\
Available & $60(51.7)$ \\
Not available & \\
Pathologic slides & $96(82.8)$ \\
Available & $20(17.2)$ \\
Not available &
\end{tabular}

*Sufficient: with information on location and shape; ${ }^{\dagger}$ Insufficient: without information on location and shape.

sions. It is suggested that the diagnostic accuracy of endoscopic gastric biopsy can be improved by taking biopsies from both the rim and the slough of an ulcer., ${ }^{3,5}$ Pinch biopsy was considered to the highest diagnostic yield for ulcerative or polypoid masses. ${ }^{5}$ So, the effort to target the best site is necessary to prevent a false-negative biopsy. The number of biopsy fragments seems to be sufficient, because the average count of biopsy fragments was 5.0 \pm 1.6 . The optimal biopsy number is a controversial issue. $^{6}$ American Society for Gastrointestinal Endoscopy guidelines have recommended the multiple biopsy specimens. ${ }^{5}$ The updated Sydney classification system is currently the most widely accepted guideline to classify and grade gastritis, and recommends to obtain five gastric biopsies.' Otherwise, in cases of visible lesions, target biopsies were essential for the diagnosis rather than multiple biopsy in another study. ${ }^{8}$ Our results suggested that diagnostic accuracy of endoscopic gastric biopsy in ulcerating lesions can be improved with target biopsy, not the large number of biopsies recommended in early studies. ${ }^{4,9}$

If the lesion is difficult to point out in endoscopic examina- tion after referral (Fig. 1, indefinite lesion group), sufficient high-quality information about the lesion must be prepared in order to improve the rate of positive biopsies. In the present study, the rate of inadequate information about the endoscopy provided by referring physicians was unacceptably high (Table 1). Recently, a high-technologic referral system was introduced as results from the difficulty to track and review poor quality information recorded by handover. ${ }^{10,11}$ This system is one of the efforts to decrease problems including illegibility and repetitive collecting of information between the referring and receiving hospitals. Though not an advanced referring system tool, effective communication between the referral and receiving physicians needs well-organized documentation of the endoscopy report, representative images and valuable pathology slides.

At the referred hospitals, review of outside pathologic slides is sometimes required. As shown in this study, a difference in diagnostic interpretation on the same patient's specimen is problematic because clinical management plans can be changed by different diagnosis. Although the Vienna classification was introduced to reduce diagnostic discrepancies, there have been discrepancies in the morphological interpretation about gastric epithelia. ${ }^{12,13}$ In the present study, 23 cases (24\%) of pathologically proven gastric cancers at previous hospital were reported to be high-grade dysplasia or no evidence of malignancy in our pathologist's review. This proportions corresponds with the results of earlier large study $(28 \%))^{14}$ In the final pathology of 23 cases, 13 cases were cancer and others were high-grade dysplasia $(n=3)$, no evidence of cancer $(n=6)$, and follow up loss $(n=1)$. Based on the final diagnosis, we thought that there were some false-positive cases in the previous pathologic interpretation.

Our study has some limitations. First, it was retrospective analysis with a database from one physician's experience. Second, there is no information about the number of gastric cancer patients seen by a single physician. Therefore, we have no information for overall rate of negative biopsy at our first endoscopy after referral for biopsy-proven gastric cancer. However, this is the first study that reviewed and analyzed negative biopsy re- 
sults in pathologically diagnosed gastric cancer.

In conclusion, negative biopsy after referral for biopsy-proven gastric cancer is mainly caused by mistargeting and lack of sufficient information during referral. When patients with biopsyproven gastric cancer are referred, detailed information with representative pictures and pathologic slides can are mandatory for accurate endoscopic and pathologic diagnosis

\section{CONFLICTS OF INTEREST}

No potential conflict of interest relevant to this article was reported.

\section{REFERENCES}

1. Jung KW, Park S, Won YJ, et al. Prediction of cancer incidence and mortality in Korea, 2012. Cancer Res Treat 2012;44:25-31.

2. Kim Y, Jun JK, Choi KS, Lee HY, Park EC. Overview of the National Cancer screening programme and the cancer screening status in Korea. Asian Pac J Cancer Prev 2011;12:725-730.

3. Hatfield AR, Slavin G, Segal AW, Levi AJ. Importance of the site of endoscopic gastric biopsy in ulcerating lesions of the stomach. Gut 1975;16:884-886.

4. Misumi A, Mori K, Ikeda T, et al. Evaluation of fibergastroscopic biopsy in the diagnosis of gastric cancer: a study of 339 cases. Gastroenterol Jpn 1978;13:255-263.

5. Faigel DO, Eisen GM, Baron TH, et al. Tissue sampling and analysis. Gastrointest Endosc 2003;57:811-816.
6. Graham DY, Schwartz JT, Cain GD, Gyorkey F. Prospective evaluation of biopsy number in the diagnosis of esophageal and gastric carcinoma. Gastroenterology 1982;82:228-231.

7. Dixon MF, Genta RM, Yardley JH, Correa P. Classification and grading of gastritis. The updated Sydney System. International Workshop on the Histopathology of Gastritis, Houston 1994. Am J Surg Pathol 1996;20:1161-1181.

8. de Vries AC, Haringsma J, de Vries RA, et al. Biopsy strategies for endoscopic surveillance of pre-malignant gastric lesions. Helicobacter 2010;15:259-264.

9. Wang TD, Van Dam J. Optical biopsy: a new frontier in endoscopic detection and diagnosis. Clin Gastroenterol Hepatol 2004;2:744753.

10. Choo MC, Thennakon S, Shapey J, Tolias CM. A web-based referral system for neurosurgery: a solution to our problems? Br J Neurosurg 2011;25:384-387.

11. Bhabra G, Mackeith S, Monteiro P, Pothier DD. An experimental comparison of handover methods. Ann R Coll Surg Engl 2007; 89:298-300.

12. Kim JM, Cho MY, Sohn JH, et al. Diagnosis of gastric epithelial neoplasia: dilemma for Korean pathologists. World J Gastroenterol 2011;17:2602-2610.

13. Kushima R, Kim KM. Interobserver variation in the diagnosis of gastric epithelial dysplasia and carcinoma between two pathologists in Japan and Korea. J Gastric Cancer 2011;11:141-145.

14. Vieth M, Görgens D, Langner C. Pseudoneoplastic regeneration of the stomach: histopathology and differential diagnosis. Pathologe 2010;31:171-176. 